nalisierung der Analyse des Präventionsdispositivs wird im nächsten Teil verdeutlicht.

\title{
3 Operationalisierung der Dispositivanalyse
}

Werden nun die bisherigen Ausführungen zum Hintergrund des DispositivBegriffs bei Michel Foucault sowie zu seiner späteren wissenschaftlichen Rezeption zusammengeführt, so ergeben sich daraus folgende Hinweise für die Untersuchung des Präventionsdispositivs.

\subsection{Analyseschritte}

Die Analyse des Präventionsdispositivs besteht aus folgenden Schritten:

- Erstens besteht das Dispositiv der Prävention aus mehreren Elementen, und zwar - hier nach Bührmann und Schneider (2012) - den Diskursen, den nichtdiskursiven Praktiken, den Vergegenständlichungen und den Subjektivationen (s. Abb. 1).

- Zweitens bestehen zwischen diesen Elementen wechselseitige Beziehungen, d.h. dass die Elemente aufeinander verweisen und ein mehr oder weniger homogenes Ensemble bilden. Diese müssen entsprechend rekonstruiert bzw. während der Analysen verdeutlicht werden.

- Drittens, dieses dispositive Ensemble existiert nicht bloß in Raum und Zeit, sondern ist in komplexe Zusammenhänge und soziale Wandlungen eingebunden und hat auch dementsprechend unterschiedliche Wirkungen und (Neben-)Effekte. Die gesellschaftstheoretische Kontextualisierung des Präventionsdispositivs muss es daher in die gegenwärtigen Entwicklungen und gesellschaftlichen Wandlungen einbetten, damit es intelligibel und zugänglich wird.

- Viertens und in Bezug auf das Zentrum der Dispositivanalyse - der Diskursanalyse - stellt das Dispositiv der Prävention eine Erweiterung des Präventions-Diskurses in dem Sinne dar, als es nicht nur »das NichtDiskursive, das Gegenständliche, das (praktische) Tun als solches« pointiert (Bührmann \& Schneider 2012: 93), sondern zu der Auseinandersetzung mit der Frage zwingt, »was aus diskursiv vermittelten Wissensordnungen inwieweit >wirk-liche ( (und insofern >machtvolle`) Effekte zeitigt, als dass es in seiner kollektiven wie individuellen Vermittlung im Selbst- wie Weltbezug handlungswirksam wird und dadurch (erst) auf jene Wissensordnungen rückwirken kann.« (ebd. [Herv. i. O.]) Eine solch erweiterte Analyse richtet sich dann auf die Ermittlung von Tatsachen, die diskursiv erzeugt wurden und 
individuell wie kollektiv gelebt sind, d.h. das, was normal empfunden und gelebt wird, in seinem Wissens- und Machtregime zu zeigen.

Abbildung 1: Schritte der Dispositivanalyse

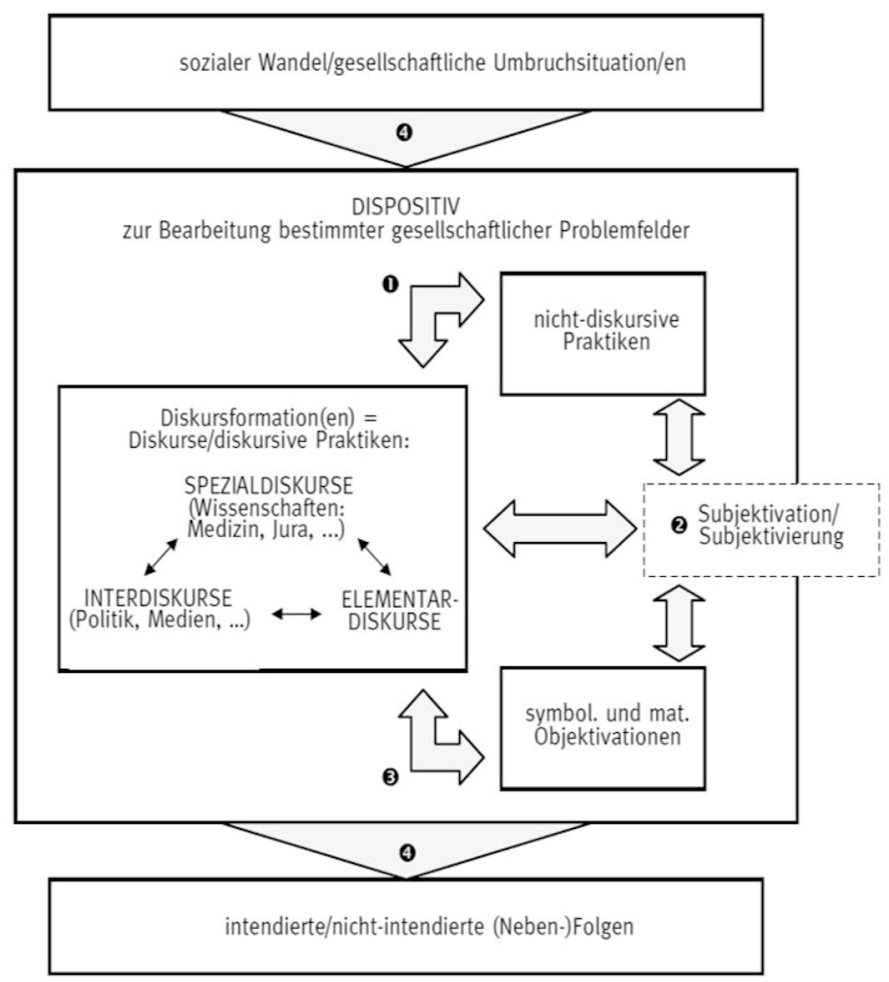

Quelle: Bührmann \& Schneider 2012: 94

Wie aus der Lektüre von Foucaults Schriften hervorgeht, bilden die Dispositive ein bestimmtes Oberflächennetz, auf dem sich seine unterschiedlichen Elemente »in einigen großen Wissens- und Machtstrategien miteinander verketten." (Foucault [1976] 1977: 128) Diesen Strategien von Kräfteverhältnissen (vgl. Foucault [1977] 1978c: 123) unterliegt eine inhärente (strategische) Intention (vgl. Diaz-Bone \& Hartz 2017: 9), die darüber entscheidet, wer die Verfügungs-Macht bzw. den $\mathrm{Zu-}$ gang zu den Führungsoptionen hat (vgl. Link 2007: 220).

Dabei entstehen Dispositive »weder zufällig, noch sind sie intentional oder von abstrakten, allgemeingültigen gesellschaftlichen Ursache-WirkungsZusammenhägen bedingt, sondern sie antworten mit einer >strategischen Zielset- 
zungく auf eine historisch spezifische Situation." (Bührmann \& Schneider 2012: 53 [Herv. i. O.]) Die Analyse solcher Strategien »ohne dahinter stehenden Strategen« (ebd.) soll die sichtbaren Kämpfe um Vorherrschaft um das erweitern, "was als Erfahrungs-Zusammenhang die Selbst-Verhältnisse von Subjekten und ihre Beziehungen untereinander als je historisch spezifische konstituiert und formiert « sichtbar macht (Foucault 1978: 132ff zit.n. Bührmann \& Schneider 2012: 54). Und an der Stelle, wo die Kräfteverhältnisse in ihren Fluchtlinien, den sich zu sich selbst verhaltenden Subjekten (vgl. Diaz-Bone \& Hartz 2017: 14) sichtbar werden, findet auch die Möglichkeit zum Widerstand und Machtumsturz statt (vgl. Link 2007: 236).

\subsection{Widerstände im Dispositiv analysieren}

Des Weiteren gilt es zu beachten, dass die dispositiven Führungsstrategien einen bereits vorkalkulierten Widerstand beinhalten und somit ihr eigenes inneres Leben entfalten, wie dies Siegfried Jäger am Beispiel des Krankenhauses verdeutlicht hat (vgl. Jäger 2015: 116). Gleichzeitig sperrt sich das Dispositiv gegen Veränderungen von außen und reagiert auf jede Bedrohung seines Verschwindens, wobei die Weisen seines Widerstands variieren können. Demnach ko-existieren im Dispositiv drei Weisen des Widerstands:

- ein vordefinierter, als möglich dargestellter Widerstand, z.B. Die Patienten haben das Recht zur Klage

- ein Widerstand, den das Dispositiv gegen seine eigene Auslöschung leistet, z.B. Die Krankenpflege ist unhinterfragbar

- und ein Widerstand, den die disponierten Subjekte, die den Zugang zur Bestimmung der Strategie des Dispositivs nicht haben, leisten (können), z.B. Ich will mich nicht , so < behandeln lassen.

Diese drei Weisen des Widerstehens bilden drei unterschiedliche Analyseperspektiven. Die erste Perspektive untersucht die Dispositionen, die innerhalb eines Dispositivs jedem seiner Elemente zugeschrieben sind: was sagbar ist, was praktiziert werden darf und welche Instrumente dafür erlaubt werden. Die zweite Perspektive untersucht die Strategien, durch die sich das Dispositiv rechtfertigt und sich nicht in Frage stellen lassen möchte. Die dritte Perspektive fokussiert auf Inkonsistenzen, Störungen und Unterbrechungen, die die dispositive Strategie bei ihrer Anwendung erfährt: Patienten, die sich nicht nach der Anweisung verhalten, die nicht kooperieren (wollen), die schweigen. In allen drei Verfahren finden Widerstände statt, entweder in Form eines geregelten und institutionalisierten Prozesses, wo die Widerstandsmöglichkeiten von vorneherein bestimmt sind, oder als eine Verweigerung des Dispositivs, Gegenstand einer Problematisierung zu sein, 
oder aber als Unbehagen der Subjekte mit dem Versuch um ihre (Fremd-)Führung. Die drei Optionen des Widerstands zeigen zugleich, wo die Macht ihre Wirkung entfaltet und werden daher zum Teil der machtkritischen Dispositivanalyse der Prävention.

\subsection{Dispositivanalyse als Forschungsstil}

Die Dispositivanalyse wird im Rahmen der vorliegenden Untersuchung weder als eine »standardisierte Vorgabe für eine notwendige Abfolge von Untersuchungsschritten oder - phasen« (Bührmann \& Schneider 2012: 109) angewendet, noch als ein »bestimmtes (Datenerhebungs- oder Auswertungs-)Verfahren für die Analyse von diskursiven und nicht-diskursiven Praktiken, Subjektivationen und Vergegenständlichungen/Objektivationen.« (Ebd.) Vielmehr ist sie hier »als ein eigener Forschungsstil zu sehen« (ebd.), der auf den Grundlagen von Foucaults Überlegungen $\mathrm{zu}$ dispositiven Ordnungen beruht und die wissenschaftliche Erweiterung des Dispositiv-Begriffs und seine Analyseoptionen miteinbezieht. In dem folgenden empirischen Teil wird daher dieser Forschungsstil »durch die perspektivisch-programmatische Fokussierung von Forschungsfragen gekennzeichnet, die auf die Verhältnisbestimmungen zwischen Diskurs, Nicht-Diskurs, Subjektivation und Objektivation in Bezug auf sozialen Wandel zielen« (ebd.), um sich der machtkritischen Auseinandersetzung mit dem Präventionsdispositiv und seiner Wirkungen auf die Selbst-Verhältnisse der Burnout-Betroffenen zu widmen.

Gleichzeitig ist zu bemerken, dass das Dispositiv der Prävention nicht erschöpfend dargestellt werden kann. Seine Analyse richtet sich vielmehr darauf, seine Funktionsweise, seine Rationalität, gleichwie seine Macheffekte zu beschreiben. Demnach werden die jeweiligen Teile der Dispositivanalyse an konkreten Beispielen - Diskursen, Praktiken, Gegenständen - analysiert, die das Dispositiv sichtbar werden lassen. Ähnlich wie bei einer archäologischen Ausgrabung, wo die entdeckten Gegenstände schrittweise in Kontext und zueinander gesetzt werden, um eine Hypothese deuten, aber auch ändern, anpassen oder präzisieren zu können, werden auch die jeweiligen Elemente des Präventionsdispositivs auf ihren Zusammenhang und auf die Hypothese überprüft, ob das Präventionsdispositiv als neoliberale Regierungstechnologie auf die Notlage zunehmend erschöpfter Subjekte reagiert. 